\title{
Quantification of the surface roughness of quartz sand using optical interferometry
}

\author{
Ting $\mathrm{Yao}^{1 *}$, Beatrice A. Baudet ${ }^{2}$, Sérgio D.N. Lourenço ${ }^{1}$ \\ 1. Department of Civil Engineering, The University of Hong Kong, Hong Kong \\ 2. Department of Civil, Environmental and Geomatic Engineering, University College London, U.K.
}

\begin{abstract}
In comparison to the description of particle size and shape, the surface roughness, which mainly affects the inter-particle friction, is more difficult to measure and quantify. One difficulty arises from the variability between particles and the heterogeneity of roughness within one particle. In this study, optical interferometry, which has the advantage of non-contact measurements of the particle surface, was adopted to measure the surface roughness of a quartzitic sand (Leighton Buzzard sand - LBS). The roughness was determined as the root mean square deviation $\left(\mathrm{RMS}_{\mathrm{f}}\right)$ of the surface from the mean plane over a field of view of $106.6^{*} 106.6 \mu \mathrm{m}^{2}$. This size of field of view is limited compared to the whole surface area of one particle. Three fractions of LBS particles were used to study the effect of particle size on the surface roughness and the roughness was measured at different points across the surface of coarse particles to assess the number of measurement points required for surface roughness quantification. The measurements revealed the followings. (1) The roughness of LBS can be measured by optical interferometry, mainly due to the high reflectivity of the quartz and the rounded particle shape. (2) $\mathrm{RMS}_{\mathrm{f}}$ of LBS with different particle sizes increases with the size of field of view first and tends to converge at larger sizes. (3) Surfaces of medium size $(1.18-2.36 \mathrm{~mm})$ particles are the smoothest. (4) Roughness of one particle varies at different measurement points, with no correlation between the mean value of $\mathrm{RMS}_{\mathrm{f}}$ and the number of points measured, while the standard deviation reaches a constant value only after a specific number of measurement points, 3 for $1.18-2.36 \mathrm{~mm}$ particles and 5 for $2.36-5 \mathrm{~mm}$ particles.
\end{abstract}

Keywords: surface roughness; optical interferometry; quantification; root mean square 


\section{Introduction}

Particle shape has a significant influence on the macro-mechanical behaviour of granular materials. Barret [1] claimed that shape should include every aspect of external morphology which comprises form, roundness and surface texture (i.e. roughness). Surface roughness mainly influences the solid-fluid interaction including fluid flow rate through rock joints [2], colloid retention [3], mineral dissolution and precipitation [4]. Brown [2] demonstrated that the surface roughness of natural rock could decrease the actual fluid flow rate by $20 \%$. Because of the discontinuous feature of rock surfaces, Fischer and Lüttge [5] proposed the concept of "converged" surface roughness parameters to quantify the surface alteration during weathering and to study the influence of surface roughness on the deposition of colloids, oxidative weathering and early-diagenetic grain coats [6-8]. For granular materials, the surface roughness has been found to have a significant effect on small-strain stiffness, shear modulus and inter-particle friction angle in both experimental studies [9-13] and DEM simulations [14-17]. In those experimental work, the tested materials were mainly artificial such as steel balls and glass beads, having uniform surface properties. However, surfaces of natural sand particles differ from those of artificial materials, due to the much more complicated formation process. Meanwhile, because of the practical difficulty in measuring accurately the surface roughness of soil grains, the quantitative characterization remains limited.

In this study, optical interferometry, which has been proved to enable the measurement of soil grain surface roughness successfully [18-23], was used on Leighton Buzzard sand (LBS). As a non-contact technique, during the measurement, the apparatus does not alter the surface of the particle. The roughness was quantified by the root mean square deviation $\left(\mathrm{RSM}_{\mathrm{f}}\right)$ of heights of discrete points within the field of view. Three different fractions of LBS particles were used to investigate the particle size effect on the surface roughness. The size of field of view used is $106.6^{*} 106.6 \mu \mathrm{m}^{2}$, which is rather small, comparing to the surface area of a sand particle. Therefore, in addition, roughness was measured at different points across the surface of coarse particles to study the variation of roughness among different measuring points in one particle, which has not been investigated in previous studies. 


\section{Experiments}

\subsection{Equipment}

Fig.1 shows the working principle of optical interferometry [24]. A beam splitter is used to divide the light beam from the light source into two beams, one will be reflected by the rough surface and the other one will be reflected by the reference mirror. The CCD camera can generate the $3 \mathrm{D}$ surface by the interference induced by two different reflected light beams. The lateral resolution is $0.184 \mu \mathrm{m}$ and the vertical resolution can be as low as $0.01 \mu \mathrm{m}$. In this study, white light interferometry was used on an optical microscope (Fogale-Nanotech, Nîmes, France). FOGALE Pilot 3D software and FOGALE Viewer 3D software were used to obtain and analyse the data.

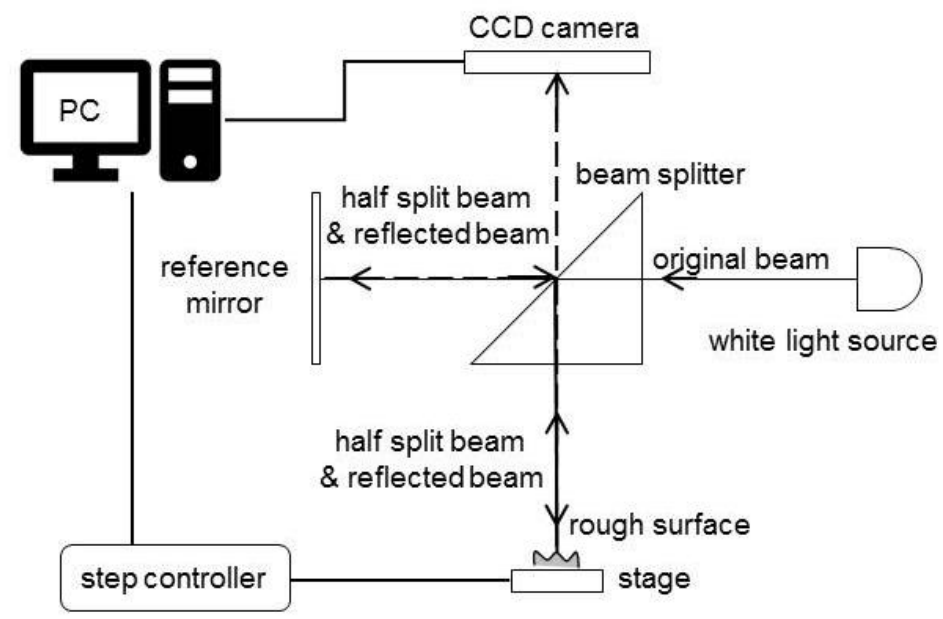

Fig.1 Schematic diagram of the optical interferometry measuring principle (Redrawn from Fogale [24])

\subsection{Particle selection and preparation}

LBS particles with sizes of $0.6-1.18,1.18-2.36$ and $2.36-5 \mathrm{~mm}$ obtained from standard sieving test [25] were selected to study the effect of particle size on surface roughness. Particle size and shape were first quantified by QicPic ${ }^{\mathrm{TM}}$ (Sympatec $\mathrm{GmbH}$, Clausthal-Zellerfeld, Germany) laser scanner which records the outline of particles falling under gravity. Fig. 2 shows some binary images of selected sand particles of three sizes with size and shape parameters, in which EQPC is the diameter of the circle with equivalent projection area as the particle; Feret diameters are the distances between two parallel line tangents to the outline of a particle image at any direction (FERET_MAX and FERET_MIN are the maximum and minimum distances respectively); sphericity is the ratio between the perimeter of the circle with equivalent projection area as the particle and the real perimeter of the projection of the particle, which equals the degree of circularity proposed by Wadell [26]; convexity is 
the ratio between the actual area and the convex hull area, which is similar to ISO solidity [27], and aspect ratio is the ratio between FERET_MIN and FERET_MAX.

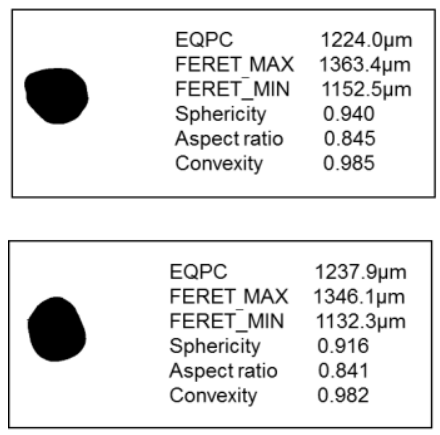

$0.6-1.18 \mathrm{~mm}$
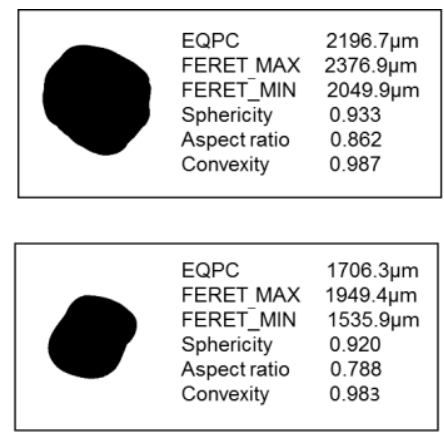

$1.18-2.36 \mathrm{~mm}$

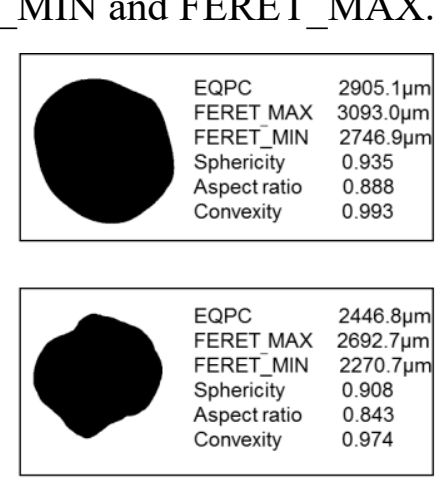

2.36-5.0mm

Fig.2 Images of selected particles from QicPic with size and shape parameters

For each size, fifty particles were randomly chosen to measure the surface roughness at one point for each particle. The results of mean values of particle size and three shape parameters are shown in Table1. The high values for the shape descriptors indicate that the selected particles have a spherical and rounded shape, which helps to minimise the influence of particle shape on the characterization of the roughness results. Before testing, the surfaces of the particles were carefully cleaned using alcohol. Comparing to one-point measurement, it is much more difficult and time consuming to conduct multiple-points measurement on one particle. In this work, another twenty LBS particles were chosen to study the variation of roughness at different points of one particle, assessing the suitability to use the roughness from one measurement point to represent the roughness of the whole particle. Only larger particles, with sizes of 1.18- $2.36 \mathrm{~mm}$ and 2.36-5 mm, were used for this purpose (ten particles for each fraction), since it is almost impossible to measure multiple points for very fine LBS particles. The number of points tested for each particle varies from 3 to 5 for smaller particles and from 3 to 9 for larger particles.

Table 1 Summary of particle size and shape parameters for LBS

\begin{tabular}{ccccc}
\hline Particle size $/ \mathrm{mm}$ & $\mathrm{D}_{\text {mean }} / \mathrm{mm}$ & Aspect ratio & Sphericity & Convexity \\
\hline $0.6-1.18$ & 1.10 & 0.791 & 0.910 & 0.975 \\
$1.18-2.36$ & 2.00 & 0.821 & 0.912 & 0.984 \\
$2.36-5$ & 2.53 & 0.787 & 0.885 & 0.975 \\
\hline
\end{tabular}




\section{Results and discussion}

3.1 Roughness measurement and quantification

Fig. 3 shows the image of an LBS particle obtained from the optical interferometry. Due to the high reflectivity and relatively regular shape of the LBS particle, the amount of invalid pixels (green dots) within the field of view can be less than $1 \%$, which guarantees the accuracy of the experimental data.

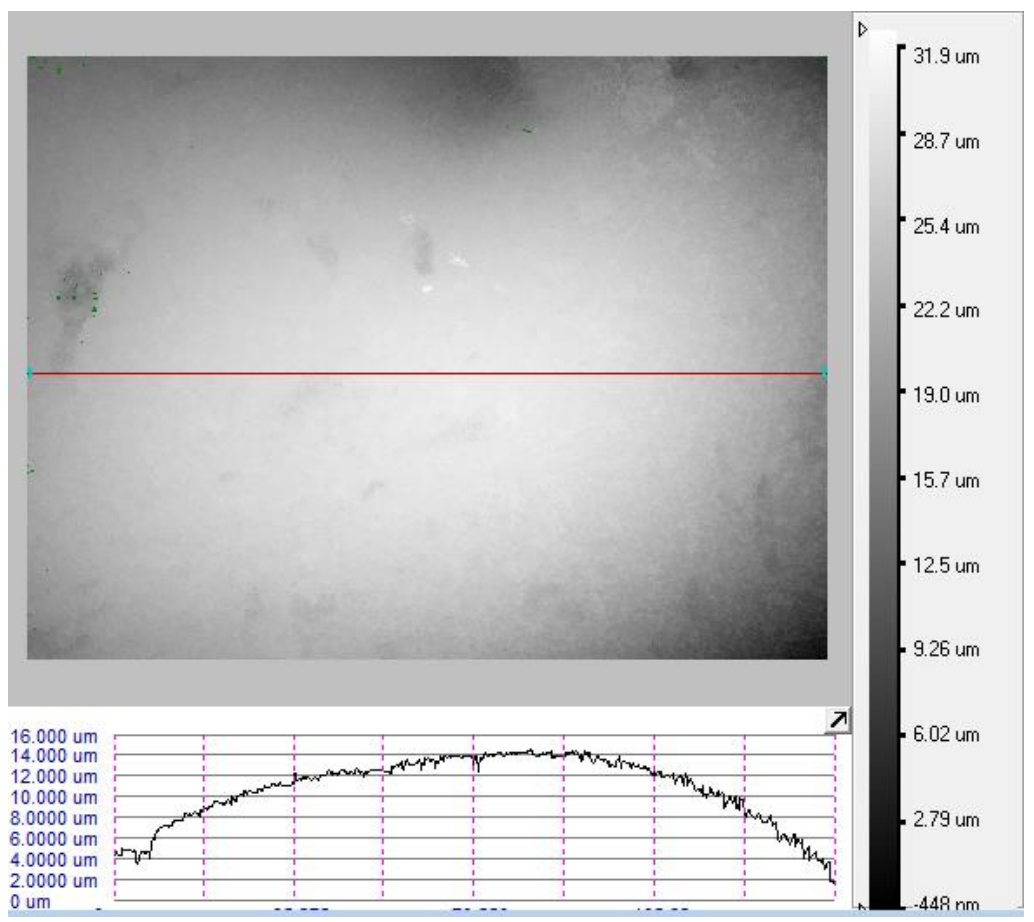

Fig.3 Roughness profile of an LBS particle from optical interferometry

As surfaces of sand particles are not flat, the finer the particles, the greater the surface curvature within the same size of field of view. Hence, a separation method is needed to remove its influence on the quantification of surface roughness. The shape motif method [28] was used to separate the whole surface into shape and roughness as shown in Fig.4. The concept of motif refers to the filtering of a surface profile between regular and irregular properties related to roughness. Although this method has the limitation that different scales were used to fit the shape of sand grains with different diameters [23], the relatively low error comparing to Yang et al.'s method and its convenience (built in the FOGALE Viewer 3D software) were the main reasons to use it in this study. After flattening, the surface roughness was quantified by $\mathrm{RMS}_{\mathrm{f}}$, which is only related to the roughness, can be calculated using Eq.1.

$$
R M S_{f}=\sqrt{\frac{1}{X Y} \sum_{i=1}^{X} \sum_{j=1}^{Y}\left(h^{2}(i, j)\right)}
$$


Where $\mathrm{X}, \mathrm{Y}$ are the numbers of points along the $\mathrm{X}, \mathrm{Y}$ directions; $\mathrm{h}(\mathrm{i}, \mathrm{j})$ is the height of discrete point to the mean plane. In this study, the measurement area is $106.6 * 106.6$ $\mu \mathrm{m}^{2}$, and the number of points along each direction is 578 (total number $578 \times 578$ ).
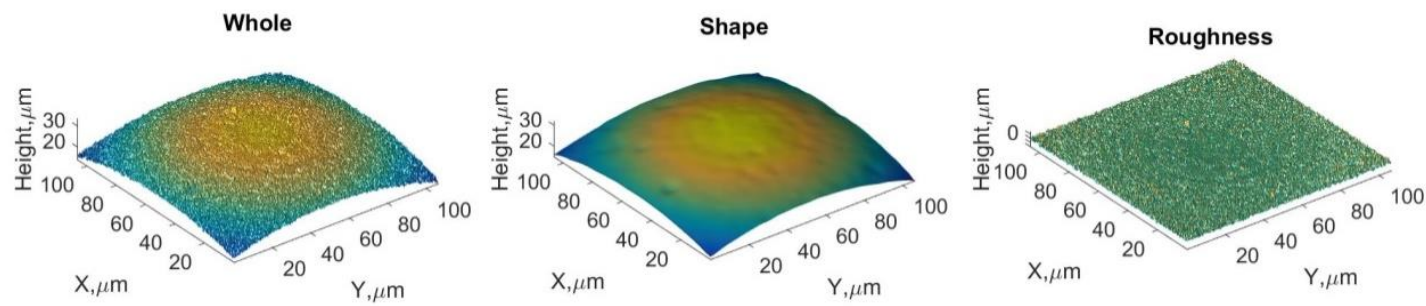

Fig.4 Shape motif method separating whole surface into shape and roughness.

\subsection{Size of field of view}

Fischer and Lüttge [5] found that the surface roughness parameters of rock tends to converge within a particular range of size of field of view, because of the composition of rock surfaces which includes mineral aggregates and pores. The tested LBS particles are made up of a single mineral $\left(\mathrm{SiO}_{2}\right)$ and thus the shape motif method was used. Therefore, the variation of surface roughness due to various mineralogies and depressions (large pores) should be limited. Cavarretta [22] and Otsubo et al. [29] both reported that the surface roughness of glass beads increases with the increasing size of field of view and pointed out that it is important to state the surface roughness data together with the size of field of view.

Similar analysis has been conducted to study the effect of size of field of view on the $\mathrm{RMS}_{\mathrm{f}}$ of LBS particles. The $\mathrm{RMS}_{\mathrm{f}}$ was first calculated for the entire data set and then subsequently for increasing smaller parts of this data set. Different from the method of decreasing the size of the field of view used by Fischer and Lüttge [5], the length of the field of view $A_{n}$ was reduced at a constant ratio of $5 \%$ from the largest length $A_{0}$ $(106.6 \mu \mathrm{m})$, i.e. $A_{n}=(1-5 \% * n) * A_{0}$. The direction of convergence is towards the center as shown in Fig. 5. Note that the peak point of the tested particle, which is of significant importance in studying the contact behaviour of sand particles, was generally put in the center of field of view. Reducing the field of view in this way ensured that the peak point is always focused.

Fig. 6 shows the correlations between the size of field of view and $\mathrm{RMS}_{\mathrm{f}}$ for particles with different sizes. The $\mathrm{x}$ coordinate is the ratio between the area of the tested field of view $\left(\mathrm{A}_{\mathrm{n}}{ }^{2}\right)$ and the minimum area $\left(10.7 * 10.7 \mu \mathrm{m}^{2}\right)$. It is found that the $\mathrm{RMS}_{\mathrm{f}}$ increases with the size of field of view firstly and then tends to converge at larger 
sizes regardless of the size of particles. Therefore, it is reasonable to use $\mathrm{RMS}_{\mathrm{f}}$ obtained from the field of view of $106.6 * 106.6 \mu \mathrm{m}^{2}$ to represent the surface roughness and study the correlation between surface roughness and particle size.

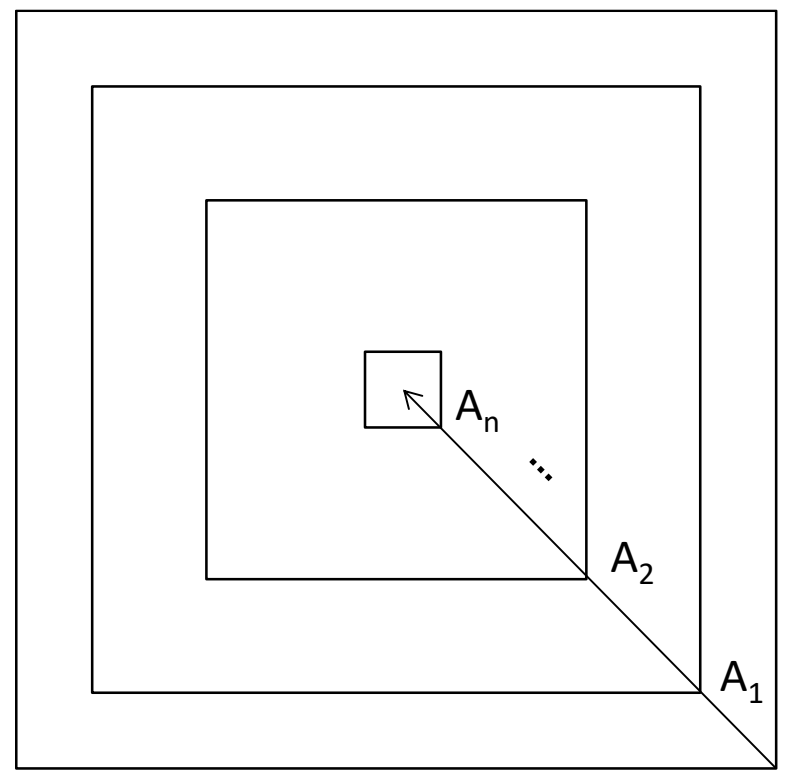

$\mathrm{A}_{0}$

Fig. 5 Reduction of size of field of view $\left(A_{0}=106.6 \mu \mathrm{m}^{2}, A_{n}=(1-0.05 * n) * A_{0}\right)$.

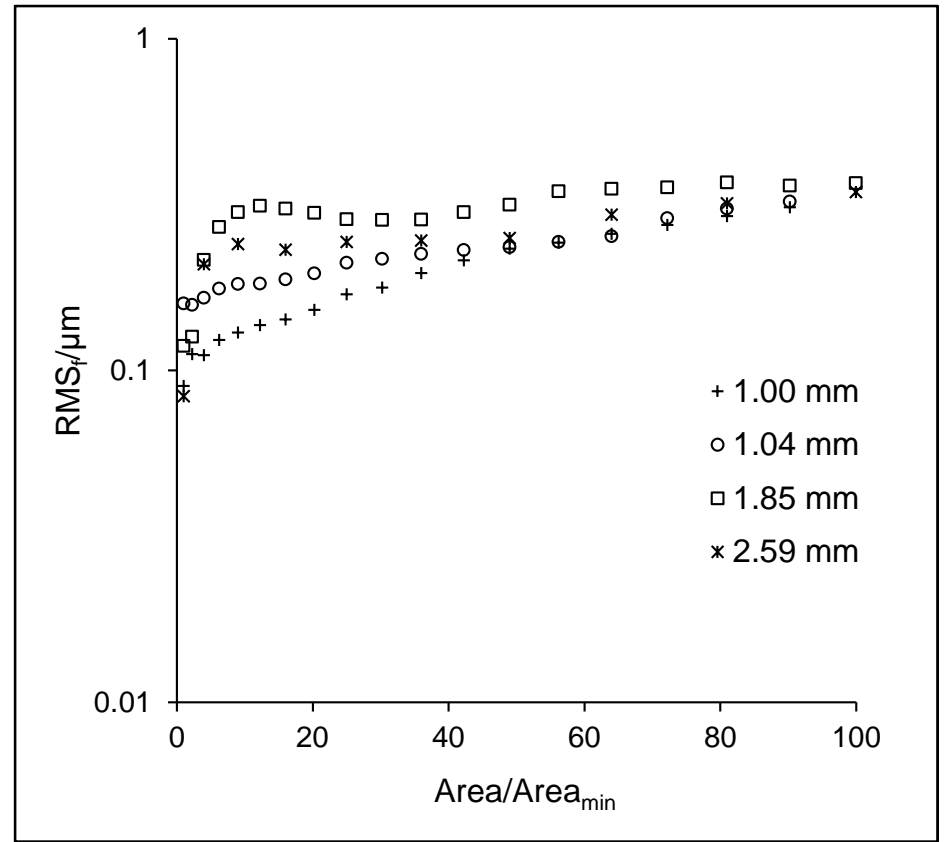

Fig.6 Effect of size of field of view on the $\mathrm{RMS}_{\mathrm{f}}$ of LBS with different particle sizes.

\subsection{Roughness of particles with different sizes}

Fig.7 shows the relationship between particle size and $\mathrm{RMS}_{\mathrm{f}}$ in terms of mean, maximum and minimum values. The results show that the mean values of $\mathrm{RMS}_{\mathrm{f}}$ 
decrease from the smallest particles $(0.60-1.18 \mathrm{~mm})$ to the medium sized particles $(1.18-2.36 \mathrm{~mm})$ and then increases slightly from the medium sized particles (1.18$2.36 \mathrm{~mm})$ to the largest particles $(2.36-5 \mathrm{~mm})$. LBS is quartzitic and chemically stable, so it is assumed that the surface roughness is mainly changed by the transportation and deposition process throughout its geological history. Two potential mechanisms control surface roughness changes. During transportation, the fluvial erosion tends to smooth the surface of LBS particles [30], while the inter-particle abrasion results in rougher surfaces for those rounded particles [20,21]. It should be noted that, the fluvial erosion is the primary effect and inter-particle abrasion works as the secondary, when those sand particles are transported in the fluvial environment [30]. The finest particles might experience both weakest fluvial erosion and interparticle abrasion because of the smallest contact area, resulted in the roughest particle surfaces. For the medium size and coarsest particles, the mean particle sizes are relatively close so that they show similar surface roughness. It is likely that the coarsest particles would experience more inter-particle abrasion due to the larger contact area, resulting in the slightly higher surface roughness. Additionally, the variation of $\mathrm{RMS}_{\mathrm{f}}$ of the medium sized particles is also the smallest, which indicates that the surface roughness of those medium sized particles is the most uniform.

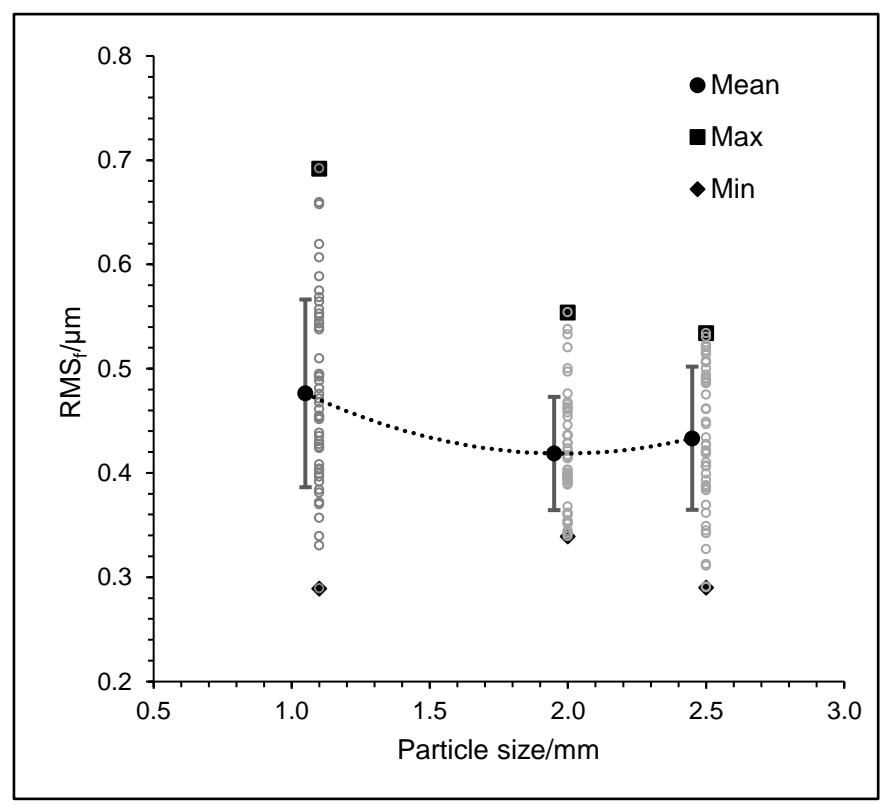

Fig.7 Effect of particle size on the $\mathrm{RMS}_{\mathrm{f}}$ of LBS

\subsection{Roughness at different points of one particle}

Fig.8 shows the local surfaces at nine different peak (i.e. convex) points of an LBS particle with a diameter of $2.5 \mathrm{~mm}$. It can be observed that the form of those surfaces 
varies, which is also assumed to be induced by different actions of fluvial erosion and inter-particle abrasion during transport.
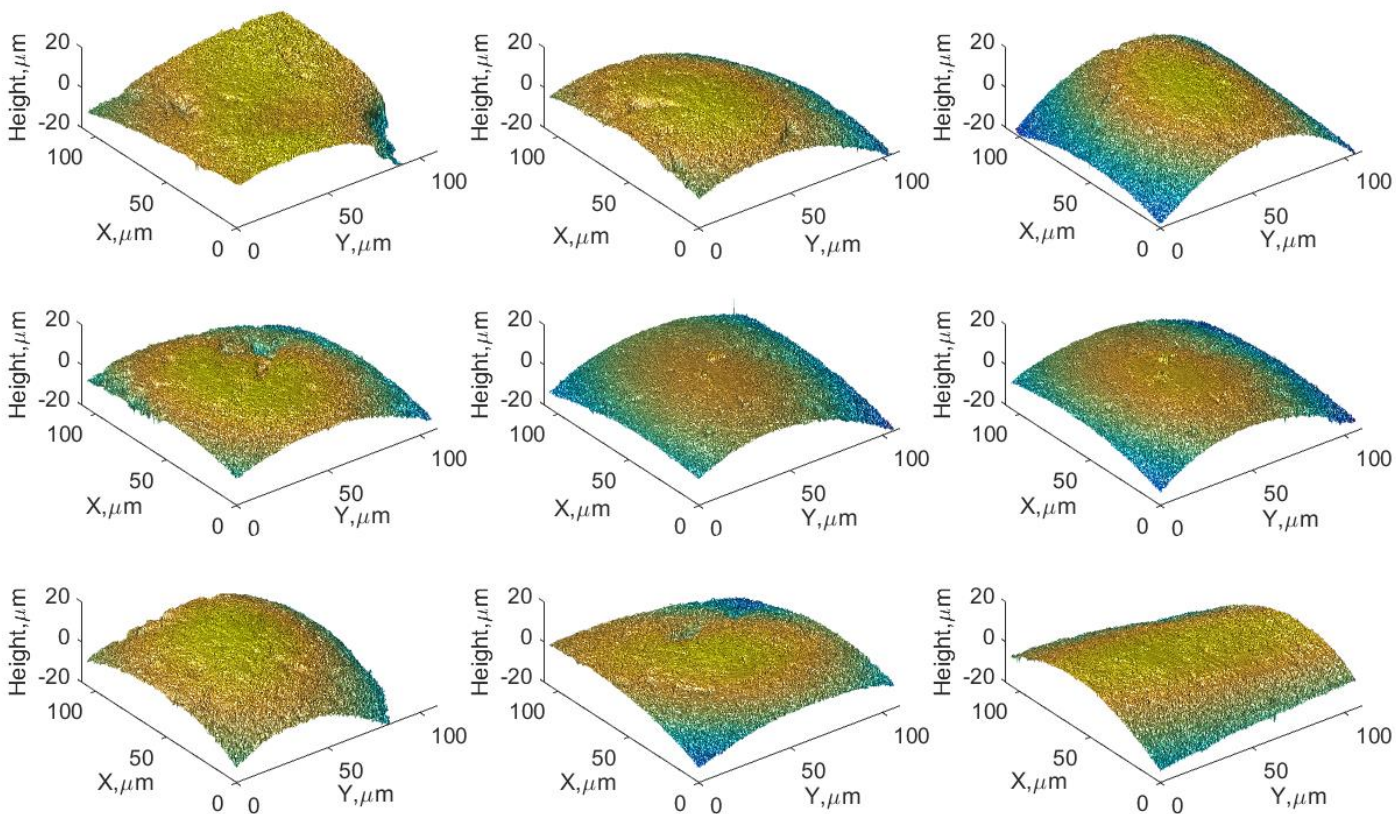

Fig.8 Nine roughness measurement points of an LBS particle

The influence of the choice of measurement points on the mean value and standard deviation of $\mathrm{RMS}_{\mathrm{f}}$ for particles with two different sizes is shown in Fig.9. The results revealed that the number of measurement points does not affect the mean value of $\mathrm{RMS}_{\mathrm{f}}$ for both sizes (see Fig.9a and 9c), which indicates that the $\mathrm{RMS}_{\mathrm{f}}$ value obtained from one measurement point can describe the average surface roughness of one particle. However, as shown in Fig.9 (b) and (d), the standard deviations of $\mathrm{RMS}_{\mathrm{f}}$ for both sizes show increasing trends, reaching constant values after 3 measurement points for the medium sized particles $(1.18-2.36 \mathrm{~mm})$ and 5 for the largest size particles $(2.36-5 \mathrm{~mm})$. This is important for the particle shape reconstruction, in which both mean value and standard deviation are required in describing the features of surface roughness. To provide more reliable surface roughness values (mean value and standard deviation), the number of measurement points should be larger than a specific value, which can be obtained from similar curves shown in Fig.9 (b) and (d).

\section{Conclusions}

Due to the high reflectivity of quartz and the spherical shape of its grains, the roughness of LBS particles can be measured by optical interferometry. $\mathrm{RMS}_{\mathrm{f}}$ of LBS 
with different particle sizes increase with the size of field of view first and tend to converge at larger sizes. By comparing the mean value and standard deviation of $\mathrm{RMS}_{\mathrm{f}}$ of particles with different sizes, it was found that the surfaces of medium sized particles are the smoothest and the most uniform, possibly because that the medium sized particles experienced more fluvial erosion but less inter-particle abrasion. Multiple points of one particle were measured to study the variation of roughness of two size fractions, $1.18-2.36 \mathrm{~mm}$ and $2.36-5 \mathrm{~mm}$. The results revealed that the roughness of one particle varies at different measurement points as expected. The number of measurement points has little influence on the mean values of roughness for two size fractions, while the standard deviation reaches a constant value only after a specific number of measurement points, 3 for 1.18-2.36 mm particles and 5 for 2.36$5 \mathrm{~mm}$ particles.
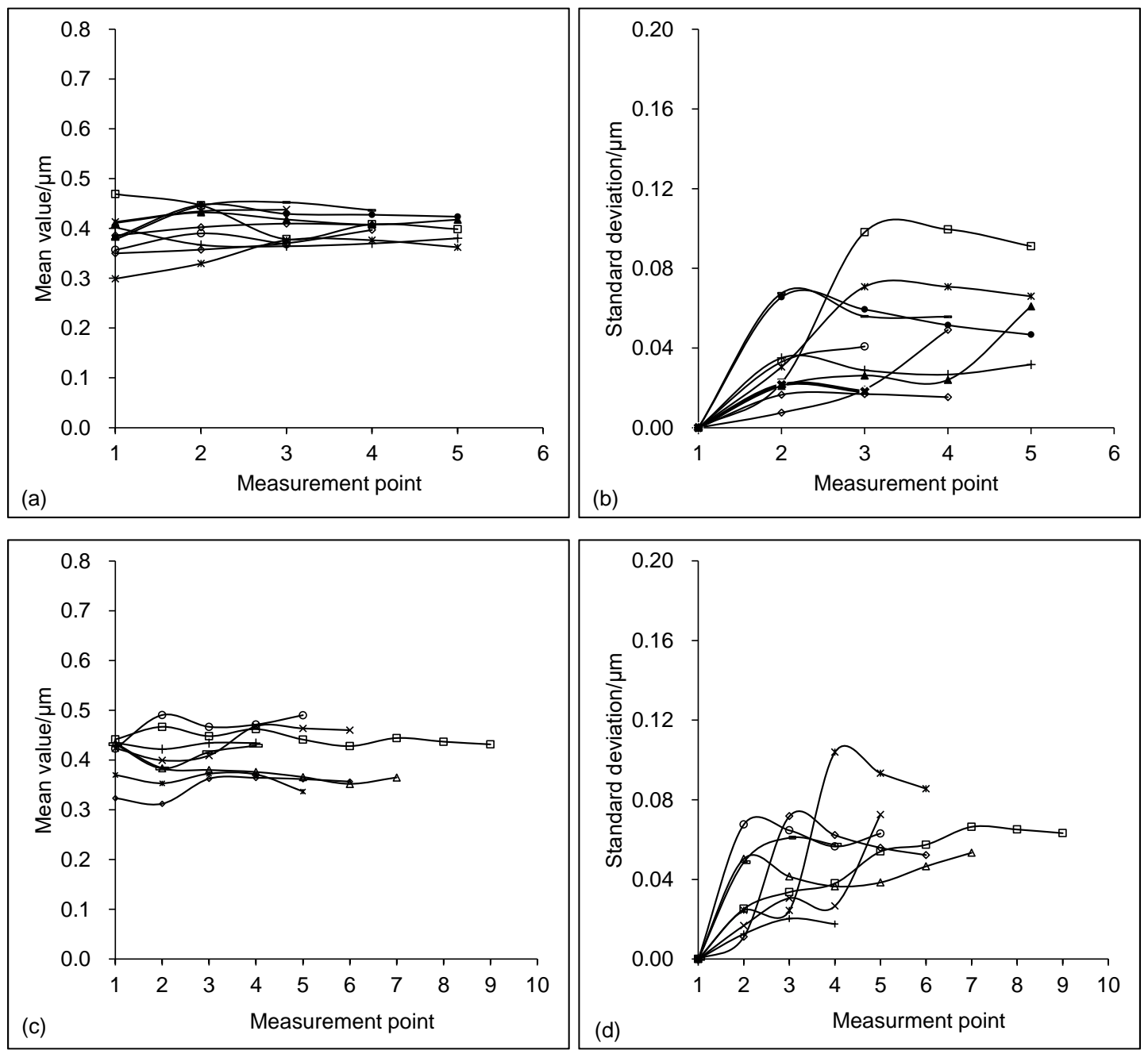

Fig.9 Effect of number of measurement points on the mean value: (a) 1.18-2.36 mm and (c); and standard deviation: (b) 1.18-2.36 $\mathrm{mm}$ and (d) 2.36-5 $\mathrm{mm}$ of $\mathrm{RMS}_{\mathrm{f}}$. 


\section{Acknowledgements}

Financial support provided by the General Research Fund, Research Grants Council, Hong Kong (17200114, T22-603/15-N) is acknowledged.

\section{References}

[1] Barrett, P.J. (1980). The shape of rock particles, a critical review. Sedimentology, 27(3), 291-303.

[2] Brown, S. R. (1987). Fluid flow rock joints: the effect of surface roughness. Journal of Geophysical Research, 92(B2), 1337-1347.

[3] Anbeek, C. (1992). Surface roughness of minerals and implication for dissolution studies. Geochimica et Cosmochima Acta, 56(4), 1461-1469.

[4] Darbha, G. K., Fischer, C., Luetzenkirchen, J. \& Schäfer, T. (2012). Site-specific retention of colloids at rough rock surfaces. Environmental Science \& Technology, 46, 9378-9387.

[5] Fischer, C. \& Lüttge, A. (2007). Converged surface roughness parameters-A new tool to quantify rock surface morphology and reactivity alteration. International Journal of Science, 307, 955-973.

[6] Fischer, C., Karius, V., Weidler P. G., \& Lüttge, A. (2008). Relationship between micrometer to submicrometer surface roughness and topography variations of natural iron oxides and trace element concentrations. Longmuir, 24, 3250-3266.

[7] Fischer, C., Michler, A., Darbha, G., Kanbach, M., \& Schafer, T. (2012). Deposition of mineral colloids on rough rock surfaces. American Journal of Science, 312: 885-906.

[8] Fisher, C., Aurin, P., Darbha, G. K., \& Arp G. (2013). Experimental approaches to the formation of early-diagenetic grain coats on quartz surfaces. German Journal of Geoscience, 164(2): 225-236.

[9] Santamarina, C. \& Cascante, G. (1998). Effect of surface roughness on wave propagation parameters. Geotechnique, 48(1), 129-136.

[10] Sharifipour, M. \& Dano, C. (2006). Effect of grains roughness on waves velocities in granular packings. In First Euro Mediterranean in Advances on Geomaterials and Structures. Hammamet, Tunisia, hal-01501211.

[11] Li, Q. \& Kim, K.S. (2008). Micromechanics of friction: effects of nanometrescale roughness. In Proceedings of the Royal Society of London A: Mathematical, Physical and Engineering Sciences, 464(2093), 1319-1343.

[12] Senetakis, K., Coop, M.R., \& Todisco, M.C. (2013). The inter-particle coefficient of friction at the contacts of Leighton Buzzard sand quartz minerals. Soils and Foundations: 53(5), 746-755.

[13] Nardelli, V., Coop, M. R., Andrade, J. E., \& Paccagnella, F. (2017). An experimental investigation of the micromechanics of Eglin sand. Powder Technology, 312, 166-174.

[14] Yimsiri, S. \& Soga, K. (2000). Micromechanics-based stress-strain behaviour of soils at small strains. Géotechnique, 50 (5), 559-571. 
[15] Soga, K. \& O'Sullivan, C. (2010). Modeling of geomaterials behavior. Soils and Foundations, 50(6), 861-875.

[16] Otsubo, M., O’Sullivan, C. \& Sim, W. W. (2014). A methodology for accurate roughness measurements of soils using optical interferometry. In Geomechanics from micro to macro, Proceedings IS-Cambridge 2014 (eds K. Soga, K. Kumar, G. Biscontin and M. Kuo), pp. 1117-1122. London, UK.

[17] Otsubo, M., O’Sullivan, C., Sim W.W., \& Ibraim, E. (2015). Quantitative assessment of the influence of surface roughness on soil stiffness. Géotechnique, 65(8), 694-700.

[18] Vallejo, L. E. (1995). Fractal analysis of granular materials. Technical note. Géotechnique, 45 (1), 159-163.

[19] Alshibi, K. \& Alsaleh, M. (2004). Characterizing surface roughness and shape of sands using digital microscopy. Journal of computing in civil engineering, 18, 31-37.

[20] Cavarretta, I., Coop, M. \& O'Sullivan, C. (2010). The influence of particle characteristics on the behaviour of coarse grained soils. Géotechnique, 60(6), 413423.

[21] Altuhafi, F. N. \& Coop, M. R. (2011). Changes to particle characteristics associated with the compression of sands. Géotechnique, 61(6), 459-471.

[22] Cavarretta, I. (2009). The influence of particle characteristics on the engineering behaviour of granular materials. PhD thesis, Imperial College, London.

[23] Yang, H., Baudet, B. A., \& Yao, T. (2016). Characterization of the surface roughness of sand particles using an advanced fractal approach. Proceedings of the Royal Society of London A, 472(2194), 20160524.

[24] Fogale (2009). Fogale nanotech user manual version 2.2.1. Nimes, France.

[25] BSI (1990). BS1377: Methods of tests for soils for civil engineering purposes. BSI, London, UK.

[26] Wadell, H. (1933). Sphericity and roundness of rock particles. Journal of Geology, 41,310-330.

[27] ISO (2006). Representation of results of particle size analysis. Part 1-6. Part 6: Descriptive and quantitative representation of particle shape and morphology. Draft International Standard ISO/DIS 9276, Geneva.

[28] Boulanger, J. (1992). An interesting complement to ISO parameters for some functional problems. International Journal of Machine Tools and Manufacture, 32(1/2), 203-209.

[29] Otsubo, M., O’Sullivan, C., \& Sim, W. W. (2014). A methodology for accurate roughness measurements of soils using optical interferometry. Geomechanics from micro to macro, Proceedings IS-Cambridge, 1117-1122.

[30] Attal, M., \& Lavé, J. (2009). Pebble abrasion during fluvial transport: Experimental results and implications for the evolution of the sediment load along rivers. Journal of Geophysical Research, 114, F04023. 